Research Article

\title{
ASD Children's APP Emotional Interaction Design Based on Smart Toys of Internet of Things
}

\author{
Bingchen Zhang, ${ }^{1}$ Yanqun Wang $\mathbb{D}^{1}{ }^{1}$ Yuling Yang, ${ }^{1}$ and Lishu Song ${ }^{2}$ \\ ${ }^{1}$ School of Mechanical and Electrical Engineering, Jiangsu Normal University, Xuzhou 221116, Jiangsu, China \\ ${ }^{2}$ Jiangsu Sheng Institute of Technology, Jiangsu Normal University, Xuzhou 221116, Jiangsu, China \\ Correspondence should be addressed to Yanqun Wang; 6020150066@jsnu.edu.cn
}

Received 31 July 2021; Revised 20 October 2021; Accepted 30 October 2021; Published 1 December 2021

Academic Editor: Sang-Bing Tsai

Copyright (c) 2021 Bingchen Zhang et al. This is an open access article distributed under the Creative Commons Attribution License, which permits unrestricted use, distribution, and reproduction in any medium, provided the original work is properly cited.

\begin{abstract}
Autism is a disorder caused by nerve developmental disorders in childhood. Autism is a serious and widespread developmental disorder, with social disorders, speech development disorders, and poor language communication skills as typical symptoms, accompanied by stereotyped behaviors. With the improvement of our country's material living standards, the frequency of childhood autism is increasing year by year. Therefore, IoT smart toys specially designed for children with autism can meet their needs and help them to carry out rehabilitation education, so that they can obtain more benefits in the process of treatment and nurturing. Smart IoT toys are not only a design that pays attention to details, but also integrates care for children with autism into the design. This article aims to research based on the Internet of Things, starting with the design of toys for children with autism. This article applies the design ideas of smart IoT toys to APP product design, introduces emotional factors into APP products, and stimulates the profound emotional experience of ASD children. Designing products from the emotional perspective and details of smart Internet of things toys and the perspective of ASD children, analyze the emotional design factors of APP, and propose application programs for ASD children according to the design standards and methods of smart Internet of things toys. After studying the status quo of children with autism, it can be concluded that children with autism need special toys suitable for them. IoT smart toys can expand the design ideas of existing children's educational toys, enrich the toy market, and provide children with more toys that meet their needs. According to the latest statistics from the US Centers for Disease Control and Prevention, from 2010 to 2014, the prevalence of children with autism in the United States rose from 0.0147 to 0.0169 , and the prevalence of children with autism aged 0-14 exceeded 200,000. With the improvement of our country's material living standards, the frequency of childhood autism is increasing year by year.
\end{abstract}

\section{Introduction}

1.1. Background of Topic Selection. Nowadays, the pathological mechanism of autism is not clear, but experts from various sources at home and abroad have believed that the disorder is a complex disorder caused by a variety of different factors. Clinically, it often manifests such as genetic, neurological, and psychological. According to research results, the disorder is not caused by the environment, and it is more likely to be congenital. Nowadays, the number of children with autism in our country is increasing year by year, but only $33 \%$ of patients are willing to receive treatment. On the one hand, the patient's family is not financially wealthy and cannot support the high cost of treatment, and on the other hand, the child's parents not paying much attention to the child's illness has only resulted in fewer and fewer people being treated. In addition, another problem of autism is that there are no drugs for treatment. In order to help autistic patients develop their self-care ability, social language, and cognitive level, the main way of treatment is education intervention, supplemented by drug therapy, and others such as diet therapy, detoxification therapy, and physical therapy. Generally, a comprehensive intervention method combining education intervention, behavior modification, and drug treatment is adopted according to the specific conditions of developmental disorders and 
emotional abnormalities of autistic patients. However, due to the emergence of smart toys for the Internet of Things, it is very convenient for children with autism to get very good emotional interaction. The Internet of Things smart toys enhance human intelligence, open the skylight of imagination, and make people smarter. It belongs not only to children but also to adults and even the elderly. Therefore, the existence of toys is very important in one's life. Modern parents have a responsibility and are willing to create a relaxed and happy environment for their children to grow up. The energy and financial resources used to develop children's intelligence and give a good education are also increasing.

1.2. Significance of the Research. Nowadays, people are paying more and more attention to autism, and various related help therapies have also received widespread attention. In the growth of children, the age of 1-6 is a period of rapid growth. If the children are treated during this period, to a large extent, the deterioration of the disorder can be inhibited and the disorder can even be cured. The smart toys based on the Internet of Things technology manufactured by this research institute can analyze the physical, psychological, cognitive, and behavioral characteristics of children with autism to a certain extent and can observe the children's behavior, aiming to help the children recover. Also, under the guidance of the Internet of Things, it will bring more emotional experience to children in the process of toy design. With the development of economy and the continuous advancement of science and technology, various electronic products have been integrated into all aspects of people's lives with their advantages such as convenience and speed [1]. It can be said that electronic mobile terminal products have been integrated with people's lives. It is closely related and inseparable. Through the study of children's cognitive habits, cognitive psychology, and usage preferences, the APP interface vision is used as the starting point to study and explore the design of human-computer interaction interface for children, which is useful for designing an APP interface that is more suitable for children. It is of great significance to fully mobilize children's interest and cultivate children's hands-on and brain-working abilities.

\subsection{Emotional Interactive APP Design Based on ASD Children.} Wang et al. believe that the application of behavioral analysis can change the abnormal behavior of autistic children very well, but this change is time-consuming and has no lasting effect [2]. Nir and Jeffrey concluded that the improvement of target behaviors in children with autism stems from social story intervention. However, in the current research process, this conclusion still lacks universality, and even some experiments cannot prove that social story intervention can effectively alleviate the behavior of children with autism [3]. Bertino et al. believe that the most natural way for children to express themselves is through play. This is a way for children to express their fantasy and explore and understand the outside world [4].

\subsection{Innovation Points of This Research}

(1) We study the design of ASD children's APP emotional interaction and adopt IoT smart toys.

(2) IoT smart toys can improve children's skills in analysis, composition, comparison, judgment, reasoning, and so on and cultivate children's depth and flexibility of thinking [5].

\section{Design Method of Emotional Interactive APP Based on ASD Children}

2.1. Concept of Emotional Interaction Design. Emotional design is the designer's use of relevant professional techniques to integrate the appearance of the product, so that the product can affect the human body's senses through sound, shape, and meaning, so that the spiritual communication between people and things can have a certain resonance. In the special product of toys for children with ASD, an emotional design is needed to make the children feel warm. Emotions are the positive or negative attitudes that a person makes in the face of external stimuli. It can describe the attitude towards surrounding things and personal behavior. When a person adds personal emotions when thinking, it will make things more complicated. Interactive design is a design that determines the behavior of man-made systems. From the user's point of view, interactive design aims to make the product easy to use and to make the whole process pleasant and efficient [6]. Interaction design can enable both parties to achieve a win-win situation. In the interaction design between the interface and the product, the connection between the user and the product can be established to meet the behavioral requirements of the controller and the supply and demand relationship between the user and the product. Game interaction design focuses on the player's behavioral interaction, so that a logical and intimate relationship between the child and the game is established, thereby affecting the child's behavior [7].

\subsection{Research Methods}

2.2.1. Literature Research Method. The literature method is to provide theoretical and mental contributions to the subsequent research of the article by reading, analyzing, and collating relevant literature materials and by comparing, analyzing, and researching a large number of domestic and foreign documents stored in the database [8].

2.2.2. Ethnographic Research. Ethnographic research is a research method for detailed description and analysis of the social and cultural life of a clear ethnic group. Studying human work and life through observation and in-depth interviews is one of the immersive research methods for researchers [9].

2.2.3. Observation Method. Observation method is the collection and perception of information by related research workers based on the research summary or observation 
table, plus various sensory organs, and the use of other auxiliary equipment to observe the research object. It has been widely used in current experiments and is a very important feature [10].

2.2.4. User Testing Method. User testing is also called product usability testing [11]. Testers must use the product to complete the general tasks assigned by the research team and record and observe the entire process of using the product.

The types of research methods are shown in Figure 1.

\subsection{Key Technologies of the Internet of Things}

\subsubsection{Radio-Frequency Identification Technology.} Radio-frequency identification technology is one of the key technologies in the Internet of Things. It can communicate data between the reader and the tag to identify the target, and it is a noncontact automatic identification system that can use radio frequency radio signals to automatically identify and acquire targets. In the smart toy, the child's signal can be identified and can be tracked and observed [12]. The system mainly includes a computer network and a reader. The electronic tag is composed of a chip and an antenna and is mainly associated with an object to identify the object, and the electronic tag has a unique electronic code to store related object information.

2.3.2. Global Positioning System. The Global Positioning System is a combination of satellite and communication technology for application [13]. GPS is an indispensable part of the Internet of Things technology. Due to its high-precision, high-efficiency, and automatic characteristics, in the development of smart toys, it cannot be affected by time and space, and can quickly, accurately, and effectively obtain the three-dimensional position, duration, and speed of the target. The development of this technology can detect the early symptoms of children with autism and lay a solid foundation for subsequent surgery.

2.3.3. Data Analysis and Processing Technology. At the architecture level of the Internet of Things, the type and amount of information transmitted from the perception layer to the application layer are gradually increasing, and the amount of data that must be processed and analyzed is doubling [14]. How to effectively mine, classify, and apply a large amount of information is a difficult problem for the Internet of Things. Data analysis and processing technology is a key part of the Internet of Things technology [15]. Data analysis can extract useful information and summarize it. Data processing is the collection, storage, retrieval, processing, transformation, and transmission of data. It is the basis on which the Internet of Things operates. Cloud computing can process tens of millions or billions of information in a few seconds, which provides a shortcut for processing a large amount of information collected from IoT systems.
2.4. Methods of Emotional Interaction Design for Smart Toys in the Internet of Things

(1) An Eye-Catching Appearance. The designer should design the appearance (shape and color) according to the characteristics of children's normal senses [16]. Because users and buyers are inconsistent, toys are special, so appearance is a problem that designers must solve, and both must be taken into consideration. Therefore, the appropriate appearance can make the game more relevant and have market power.

(2) Creative Game Play. The psychological and emotional design of children's interactive toys is a popular trend in modern toy design [17]. Interactive design is a popular design category, and emotional design is more of a creative behavior activity, allowing people to go back to the source, start from the emotions, and hit the heart directly. Combining the two together can help in developing the design. Smart toys are more popular with children and can better help them.

(3) Variability and Continuity. Various children's toys not only have a specific shape, but can also be disassembled and reorganized, thereby injecting emotion and vitality into the toy [18]. The continuity of children's toys means that the process of building friends between children and toys is expanded. In the process of playing with toys, it forms emotional nutrition [19].

\section{Experiments on the ASD Children's APP Emotional Interaction Design Research Based on the Smart Toys of the Internet of Things}

\subsection{Intervention Measures for Children with Autism}

3.1.1. Applied Behavior Analysis Therapy. Applied behavior analysis therapy is to decompose the target task into smaller tasks according to a certain method or sequence and concentrate on the task after training and decomposition in a certain period of time. After each task is completed, a certain enhanced stimulation will be given. This method can split the child's complex conditions during the treatment of children with autism and then conduct targeted treatments to provide some help to children in need. As the child's ability gradually declines, it is necessary to give the child a short and appropriate rest between these two tasks. Rehabilitation uses individual and specific methods. These methods are systematic, scientific, and rigorous and are maintained. They are strengthened to a certain extent and maintained for a certain period of time [20].

3.1.2. Sensory Integration Training Method. This means that in the same environment, the children are given a variety of stimuli (skin sensation, vision, hearing, smell, and so on) to different sensory organs (blood vessels, muscles, and joints), so as to stimulate the children's brain to process and integrate and gradually adapt to the environment [21]. 


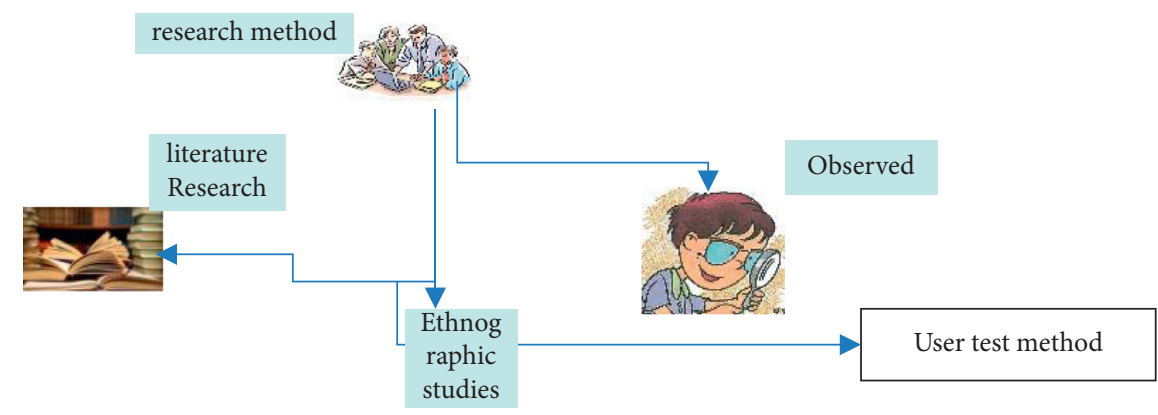

FIGURE 1: Research method type.

3.1.3. Structured Education Law. This is a method of combining certain things according to certain rules or forms to help children with autism develop normal behaviors and to help children with autism adapt to the family and society to the greatest extent [22].

3.1.4. Game Therapy. This includes observing the children's games and behaviors under natural conditions to vent the children's bad emotions, enhance the children's resistance, and promote the recovery of the disorder [23].

3.1.5. Music Therapy. Music therapy is to use music to evaluate the physical condition and mental illness of patients suffering from neurological and mental diseases or physical diseases, and to determine the goals of music therapy based on the evaluation results, so as to achieve the purpose of repair and maintenance.

\subsubsection{Interpersonal Relationship Development Intervention} Therapy. The basis of autism is that children have obstacles in understanding other people's expressions, emotions, and mental activities and sharing obstacles, leading to obstacles in interpersonal relationship development, and the use of interpersonal development intervention therapy can break the children's obstacles to a certain extent, so that children can communicate with others normally and express their feelings and ideas clearly [24].

3.1.7. Picture Exchange System Law. Using the role of reinforcers, loneliness gradually improves the communication skills and communication skills of symptomatic children in the process of structuring [25].

3.1.8. Floor Time Therapy. It is a development-oriented, systematic, and autistic intervention method that focuses on the interaction between parents and children in the family environment and emphasizes child-centered intervention methods [26].

The methods of intervention for children with autism are shown in Figure 2.

\subsection{IoT Smart Toy Model}

Input: the number of smart toys $k$ and the dataset $Y=\left\{y_{1}, y_{2}, \ldots, y_{n}\right\}$ containing $n$ objects.

Output: $k$ number $\left\{S_{1}, S_{2}, \ldots, S_{k}\right\}$ to minimize the objective function.

(i)Select the number of toys $k$.

(ii)Randomly select $k$ objects from the data as the toy center $c_{1}, c_{2}, \ldots, c_{k}$.

(iii)Assign the object $y_{i}(i=1,2, \ldots, n)$ to the nearest smart toy center $c_{j}, 1<j<k$ in turn, where $m$ is the number of attributes of the data:

$$
\left\|y_{i}-c_{j}\right\|=\min _{1<j<k} \sqrt{\sum_{l}^{m}\left(y_{i l}-c_{j l}\right)^{2}} .
$$

(iv)Calculate the new center $c_{j}$ of each smart toy, where $N_{j}$ is the number of objects contained in the $j$ th smart toy $S_{j}$ :

$$
c_{j}=\frac{1}{N_{j}} \sum_{y_{i} \in s_{j}} y_{i}, \quad j=1,2, \ldots, k .
$$

(v)If the smart toy center does not change and the objective function is the smallest, the algorithm stops [13].

3.3. Key Technology of Data Acquisition System. The relationship between the time-continuous analog signal $x(t)$ and the frequency spectrum $X(f)$ can be expressed as

$$
\begin{aligned}
x(t) & =\int_{-\infty}^{+\infty} X(f) e^{i 2 \pi f t} d f, \\
X(f) & =\int_{-\infty}^{+\infty} x(t) e^{-i 2 \pi f t} d t .
\end{aligned}
$$

The discrete sampling pulse signal $x(n T s)$ recovers the continuous signal. According to the Nyquist sampling theorem, the sampling interval of discrete pulse signal $x$ $(n T s)$ should satisfy condition $T_{s} \leq 1 /(2 f)$, and when the frequency $f_{s}$ of the sampled signal is more than twice the maximum frequency $f_{\max }$ of the sampled signal, the sampled signal can recover the sampled continuous signal, as shown in 4 and 5 ; the interval is set to $\left[-1 / 2 T_{s}, 1 / 2 T_{s}\right]$. 


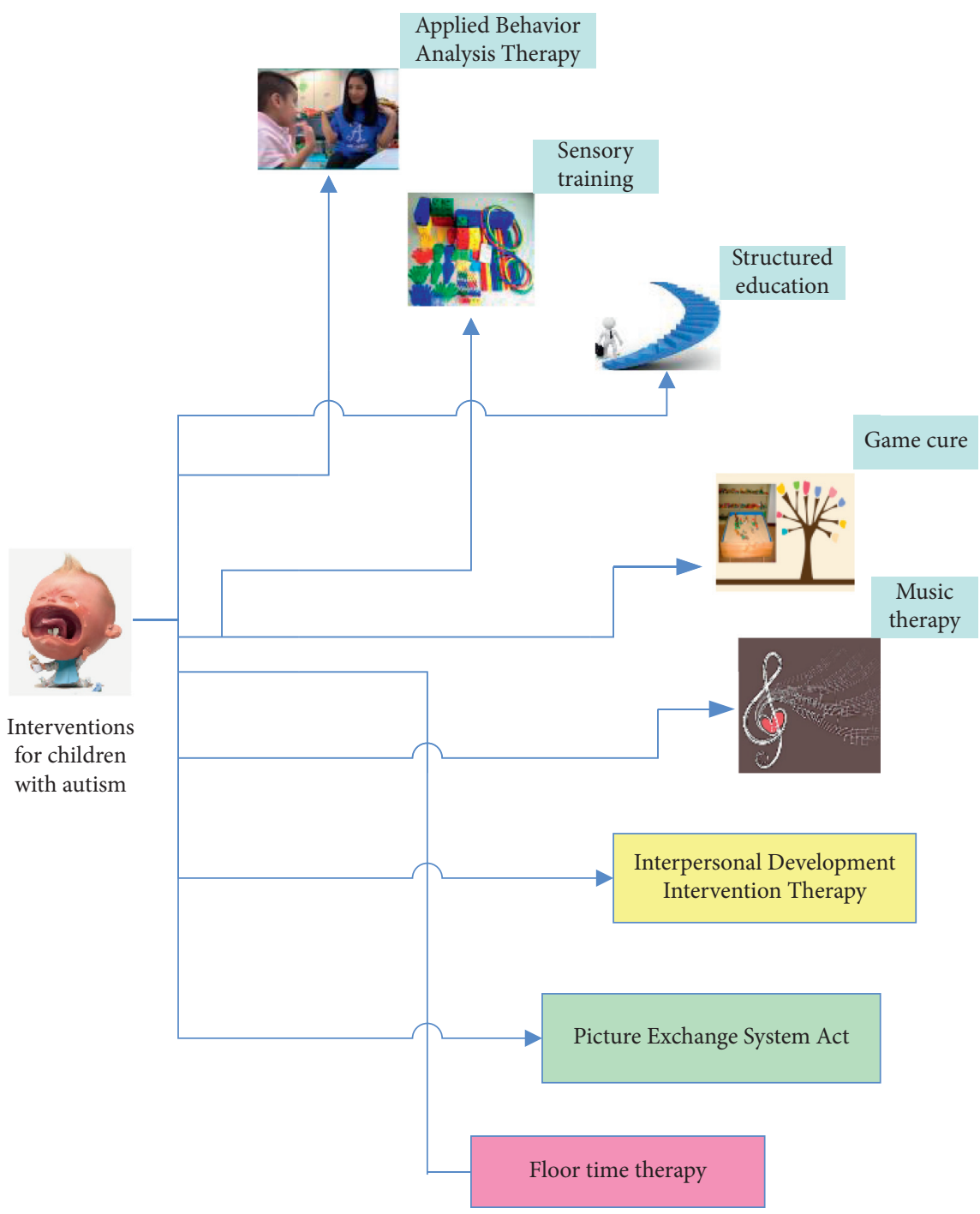

FIgURE 2: Intervention measures for children with autism.

$$
\begin{aligned}
x\left(n T_{s}\right) & =\int_{-1 / 2 T_{s}}^{1 / 2 T_{s}} X(f) e^{i 2 \pi f n T_{s}} d f, \\
X(f) & =T_{s} \sum_{n=-\infty}^{+\infty} x\left(n T_{s}\right) e^{-i 2 \pi f n T_{s}} .
\end{aligned}
$$

$x(n T s)$ can completely determine $X(f) ; x(t)$ can be determined as follows:

$$
x(t)=T_{s} \sum_{n=-\infty}^{+\infty} x\left(n T_{s}\right) \int_{-1 / 2 T_{s}}^{1 / 2 T_{s}} e^{i 2 \pi f\left(1-n T_{s}\right)} d f .
$$

\section{ASD Children's APP Emotional Interaction Design Research Based on IoT Smart Toys}

4.1. Market Research and Analysis. The preparation stage of children's smart toys is the design analysis stage. This stage requires analysis and research based on the acquired information and finds out the useful information to be used. This information can be obtained from the Internet or through your own experimental research. What we found and the preparations we made were all to lay a good foundation for the final design. Therefore, the content of design analysis includes user analysis, market analysis, and demand analysis.

(1) User Analysis. In the early stages of design, the final design direction must be determined according to the various attributes of the product user.

(2) Market Analysis. Market analysis is the analysis of product cost, sales volume, and usage, investigating market demand and formulating product development directions.

(3) Demand Analysis. The demand analysis is based on the first two analyses, and its purpose is very powerful. Starting from demand, design positioning is a common practice in all design fields. User and market demands are dynamic and need to be continuously and dynamically adjusted according to demand analysis in different time periods. 


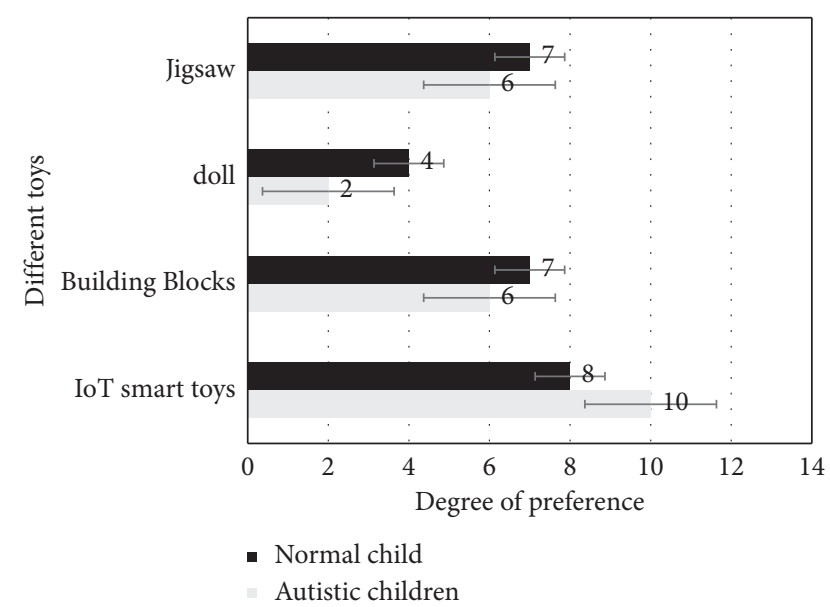

FIGURE 3: Autistic children's preference for different toys.

TABle 1: Different genders of autistic children having different color preferences for IoT smart toys.

\begin{tabular}{lccc}
\hline Children's color preference & $25 \%$ & $50 \%$ & $100 \%$ \\
\hline Boys & $27 \%$ & $19 \%$ & $54 \%$ \\
Girls & $39 \%$ & $30 \%$ & $32 \%$ \\
\hline
\end{tabular}

4.2. User Analysis of Children's Emotional Interactive Toys. The emotional design of children's interactive toys should be suitable for children's physical and psychological characteristics. The emotional process of general products is a subjective consumer experience, that is, whether the purchased goods meet their own needs, but toys have the characteristic of distinguishing consumers from users, and therefore, when buying children's toys, parents are the collectors of information and determine the main market behavior. The extent of children's influence on their parents and the impact on this behavior seem to be great. Therefore, toy design should consider not only the feeling of the buyer (parent) but also the feeling of the toy user (child).

4.3. Autistic Children's Preference for Different Toys. The degree of preference for different toys of autistic children is shown in Figure 3.

\subsection{Different Genders of Autistic Children Have Different} Color Preferences for IoT Smart Toys. Different genders of autistic children have different color preferences for IoT smart toys, as shown in Table 1.

It can be concluded that most boys prefer colors with the highest purity of $100 \%$ and prefer different purities of the same color. There is no obvious difference in girls' preferences for different purities. They prefer colors with a purity of $25 \%$.

\section{Conclusions}

With the development of economy and the continuous advancement of technology, various electronic products have been integrated into all aspects of people's lives with their advantages such as convenience and speed. It can be said that electronic mobile terminal products, smart toys, APPs, and so on have been closely related to people's lives and are inseparable. This article studies the emotional interaction design of IoT smart toys from the perspective of children's cognitive development and follows the characteristics of smart early education toys, the interaction interface model, and the information interaction model of general smart products. We constructed intelligent early education toys and analyzed the child's interactive model. Based on the basic principles of Internet user interface design, guided by children's physical, psychological, and emotional needs, the principles of emotional visual design of children's products are constructed. Based on the principle of emotional visual design of children's products and based on the three-level theory of emotional design and the flexible use of design elements of emotional symbols, we will explore how to design children's product emotional visuals. It has helped autistic children recover well. However, China's APP interface design started late, and interface design is still relatively unfamiliar to most people. The interface design of the APP is mainly based on learning from foreign excellent cases. The product interface design with few originality is still in the exploration and learning stage. For this reason, we should design and explore from the perspective and thinking of children's interface.

\section{Data Availability}

No data were used to support this study.

\section{Conflicts of Interest}

The authors declare that they have no conflicts of interest.

\section{Acknowledgments}

This study was supported by the MOE (Ministry of Education of the People's Republic of China) Project of Humanities and Social Sciences, China (grant no. 18YJAZH123), and the Natural Science Foundation of the Jiangsu Higher Education Institutions, China (grant no. 19KJD460004).

\section{References}

[1] P. Wang and W. Han, "Construction of a new financial E-commerce model for small and medium-sized enterprise financing based on multiple linear logistic regression," Journal of Organizational and End User Computing, vol. 33, no. 6, pp. 1-18, 2021.

[2] G. Wang, M. Atiquzzaman, and Z. Yan, "Security, privacy, and anonymity in computation, communication, and storage," A Framework for Preventing the Exploitation of IoTSmart Toys for Reconnaissance and Exfiltration, vol. 10658, pp. 581-592, 2017, Lecture Notes in Computer Science.

[3] K. Nir and V. Jeffrey, "Cyberthreats under the bed," Computer, vol. 51, no. 5, pp. 92-95, 2018.

[4] E. Bertino, K.-K. R. Choo, D. Georgakopolous, and S. Nepal, "Internet of things (IoT)," Acm Transactions on Internet Technology, vol. 16, no. 4, pp. 1-7, 2016. 
[5] H. Gul, N. Erol, D. Pamir Akin et al., "Emotional availability in early mother-child interactions for children with autism spectrum disorders, other psychiatric disorders, and developmental delay," Infant Mental Health Journal, vol. 37, no. 2, pp. 151-159, 2016.

[6] T. U. Khotyleva and S. A. Rosenblum, "Illusions of inclusion: typical mistakes of parents of ASD children. Inclusive school experience," Autism and Developmental Disorders, vol. 15, no. 3, pp. 48-53, 2017.

[7] I. J. Lee, C. H. Chen, and C. P. Wang, "Augmented reality plus concept map technique to teach children with ASD to use social cues when meeting and greeting," The Asia-Pacific Education Researcher, vol. 27, no. 3, pp. 1-17, 2018.

[8] M. Magrini, O. Curzio, A. Carboni, D. Moroni, O. Salvetti, and A. Melani, "Augmented interaction systems for supporting autistic children. Evolution of a multichannel expressive tool: the SEMI Project feasibility study," Applied Sciences, vol. 9, no. 15, p. 3081, 2019.

[9] M. Elhaddadi, H. Maazouz, N. Alami et al., "Serious games to teach emotion recognition to children with autism spectrum disorders (ASD)," Acta Neuropsychologica, vol. 19, no. 1, pp. 81-92, 2021.

[10] M. Yaremchuk, "The use of environmental approach in the work with children with ASD," Autism and Developmental Disorders, vol. 17, no. 4, pp. 12-20, 2019.

[11] L. B. Jahromi, K. S. Kirkman, M. A. Friedman, and A. D. Nunnally, "Associations between emotional competence and prosocial behaviors with peers among children with autism spectrum disorder," American Journal on Intellectual and Developmental Disabilities, vol. 126, no. 2, pp. 79-96, 2021.

[12] A. T. Wieckowski and S. W. White, "Attention modification to attenuate facial emotion recognition deficits in children with autism: a pilot study," Journal of Autism and Developmental Disorders, vol. 50, no. 1, pp. 30-41, 2020.

[13] B. A. Mahagamage, L. C. Rathnayake, and M. Chandradasa, "Parental perspectives on the lived experience of having a child with autism spectrum disorder in Sri Lanka," Sri Lanka Journal of Child Health, vol. 50, no. 1, pp. 110-115, 2021.

[14] L. A. Tipton, J. B. Blacher, and A. S. Eisenhower, "Young children with ASD: parent strategies for interaction during adapted book reading activity," Remedial and Special Education, vol. 38, no. 3, pp. 171-180, 2017.

[15] R. S. Bhadoria and N. S. Chaudhari, "Pragmatic sensory data semantics with service-oriented computing," Journal of Organizational and End User Computing, vol. 31, no. 2, pp. 22-36, 2019.

[16] "The diagnostic aspect of efficacy study of educational organization interactions with parents of children with ASD," Autism and Developmental Disorders, vol. 16, no. 4, pp. 35-41, 2018.

[17] G. E. Fleming, E. R. Kimonis, A. Datyner, and J. S. Comer, "Adapting internet-delivered parent-child interaction therapy to treat Co-occurring disruptive behavior and callous-unemotional traits: a case study," Clinical Case Studies, vol. 16, no. 5, pp. 370-387, 2017.

[18] L. K. Boyce, R. B. Seedall, M. S. Innocenti et al., "Influence of a parent-child interaction focused bookmaking approach on maternal parenting self-efficacy," Infants and Young Children, vol. 30, no. 1, pp. 76-93, 2017.

[19] V. Sathiyamoorthi, P. Keerthika, P. Suresh, Z. Justin, A. P. Zhang Rao, and K. Logeswaran, "Adaptive fault tolerant resource allocation scheme for cloud computing environments," Journal of Organizational and End User Computing, vol. 33, no. 5, pp. 135-152, 20221.

[20] A. Golubchikova and N. Korobtseva, "Inclusive design: systems interaction society - textile means of rehabilitation child," Bulletin of Science and Practice, vol. 6, no. 1, pp. 198-206, 2020.

[21] E. Sepahvand, H. Khankeh, M. Hosseini, and B Akhbari, "Emotional interaction in road traffic injury: a qualitative study on people with spinal cord injury," Journal of Medicine and Life, vol. 12, no. 4, pp. 419-425, 2019.

[22] I. Leppänen, R. P. Hämäläinen, E. Saarinen, and M. Viinikainen, "Intrapersonal emotional responses to the inquiry and advocacy modes of interaction: a psychophysiological study," Group Decision and Negotiation, vol. 27, no. 6, pp. 933-948, 2018.

[23] O. R. Dobrushina, L. A. Dobrynina, G. A. Arina et al., "Interaction of interoceptive perception and emotional intelligence: a functional neuroimaging study," Neuroscience and Behavioral Physiology, vol. 50, no. 8, pp. 1043-1050, 2020.

[24] N. Molly, C. H. Katie, and U. Muhammet, "PSIII-21 Relationship between companion animal interaction and emotional safety within college students," Journal of Animal ence, no. Supplement_3, p. 280, 2019.

[25] X. Liu, Y. Kawamura, T. Shimada et al., "Association of the oxytocin receptor (OXTR) gene polymorphisms with autism spectrum disorder (ASD) in the Japanese population," Journal of Human Genetics, vol. 55, no. 3, pp. 137-141, 2010.

[26] K. N. Thakkar, F. E. Polli, R. M. Joseph et al., "Response monitoring, repetitive behaviour and anterior cingulate abnormalities in autism spectrum disorders (ASD)," Brain: A Journal of Neurology, vol. 131, no. Pt 9, pp. 2464-2478, 2008. 